\title{
Significant Risk Factors for Acute Kidney Injury= A Case Control Study
}

\author{
Samiullah", Muhammad Ayub", Muhammad Saeed ${ }^{1}$, Aleem ur Rasheed ${ }^{2}$, Aminullah$^{1}$, Saddar Raheem ${ }^{3}$
}

\section{ABSTRACT}

Background: Acute Kidney injury(AKI) is one of the common diseases that's affect more than 13 million people annually. Diabetes mellitus, hypertension, upper respiratory tract infection, septic shock and nephrotoxic agents are significant risk factors for Acute Kidney Injury.

Material and Methods: This case control study was doneon 200 urinary tract infected (UTI). The inclusion criteria for cases were patients with Acute Kidney Injury age above 15 years, both genders, diagnosed urinary tract infection. Controls were similar to cases except without Acute Kidney Injury. Demographics and risk factors for Acute Kidney Injury were recorded. We use Student $t$ test compare continuous variable. For categorical variables we used Chi-square test. Multivariate logistic regression was carried using Acute Kidney Injury as dependent variable and risk factors as independent variables. $\mathrm{P}=0.05$ was considered significant.

Objectives: To determine significant risk factors for acute kidney injury.

Results: The male were $87(43.5 \%)$ and female were $113(56.5 \%)$. The mean age of the study was $45.19 \pm 16.2$ years. The frequency of diabetes mellitus $(P=.008)$, hypertension $(P<.001)$, upper respiratory tract infection $(P=.030)$, septic shock $(P=.011)$ and nephrotoxic agents $(P=.013)$ were more in cases than controls and results were statistically significant. Positive association was found for Acute Kidney Injury with increasing age $(\mathrm{OR}=1.06 ; 95 \% \mathrm{Cl}=1.017,1.105)$, serum creatinine $(\mathrm{OR}=5.9 ; 95 \% \mathrm{Cl}=2.85$, 12.55). There was negative association between glomerular filtration rate (GFR) and Acute Kidney Injury (OR=0.92; $95 \%$ $\mathrm{Cl}=0.888,0.953)$.

Conclusion: After controlling confounding factors through multivariate analysis the increasing age, serum creatinine and low GFR were significant risk factor for acute kidney injury.

Keywords: Acute kidney injury, risk factors, urinary tract infection, serum creatinine

This article may be cited as: Samiullah, Ayub M, Saeed M, Rasheed AU, Aminullah, Raheem S. Significant Risk Factors for Acute Kidney InjuryA Case Control Study. J Saidu Med Coll Swat 2021;11(2):73-77

\section{INTRODUCTION}

The classical definition of acute kidney injury (AKI) is the abrupt and sustained decrease in function of kidney. Nevertheless, the exact cutoffs diagnosis of $\mathrm{AKI}$ is not agreed. ${ }^{1,2}$ Worldwide the AKI is a common disease affecting more than 13 million people annually, $85 \%$ of whom belonged to developing countries. ${ }^{1}$ In developed countries the acute kidney injury is rarely a community-acquired disease; the primary source of AKI in these countries is the hospitalizations of patients. There is five-to-tenfold increase in the incidence of hospital-acquired acute kidney injury in these areas. ${ }^{3}$ Conversely, in less-developed countries the AKI frequently found in the community. ${ }^{4}$

One of the frequent cause of acute kidney injury is the sepsis ${ }^{5}$, and more than half of affected subjects by septic shock lead to acute kidney injury $^{6}$. Acute urinary tract infection (UTI) may lead to decline in renal function, particularly in case of urinary tract obstruction. Acute kidney injury during acute care is associated with high degree

\footnotetext{
1. Department of Medicine Saidu Medical College/Teaching Hospital Swat

2. Department of Pathology Saidu Medical College/Teaching Hospital Swat

3. Department of Paediatric Surgery Saidu Medical College/

Teaching Hospital Swat
}

Correspondence: Dr. Samiullah

Senior Registrar of Nephrology Saidu Medical College, Swat

Email: sami396@yahoo.cm

Received: February 19, 2020 Accepted: December 21, 2020 of morbidity as well as mortality. ${ }^{7}$ Patients affected by severe form of $\mathrm{AKI}$ are at more risk for end stage renal disease and even fatality during postdischarge period from the hospital. ${ }^{8}$

In developing countries the etiology of acute kidney injury are; tropical infections (e.g. leptospirosis, malaria and dengue), other form of infections (gastroenteritis, pneumonia, skin infection), acute glomerular disorders, gynecological issues, pre-disposing renal diseases (kidney, cardiac problem, diabetes mellitus, etc), herbal treatment, trauma, nephrotoxic agent, environmental causes like snake bites, and hospital-acquired AKI. Surgical intervention can lead to $\mathrm{AKI}^{9-11}$

The diagnosis of acute kidney disease can be made when glomerular filtration rate (GFR) decrease by more than $50 \%$ or two fold increase in serum creatinine comparative to baseline creatinine as per the RIFLE GFR criteria. ${ }^{12}$ Hsiao et al. ${ }^{12}$ conducted an study to know the risk factors of AKI in having UTI patients. They reported that associated risk factors for AKI were older age, upper UTI, diabetes mellitus (DM), lower baseline eGFR and a febrile during hospitalization.

There is lack of local studies on the risk factors for AKI which can different across the population due to genetic, ethnic and environmental factors. So the objective of this study was to know the risk factors for acute kidney injury. 


\section{MATERIALAND METHODS}

This case control study was done in Medical Department, of Saidu teaching hospital, Swat. A total of 200 patients (100 cases and 100 controls) were selected by consecutive sampling technique. After in depth explanation of objective and benefits of the research verbal informed consent was taken from all participants. The participants were ensured for maintaining their confidentiality.

The inclusion criteria for cases were patients with AKI, age above 15 years, both gender, diagnosed urinary tract infection cases (pain on micturition, lumbar aches or Patient is febrile and having more than 104 colony forming units $/ \mathrm{mL}$ of bacterial isolation .Controls were similar to cases except without AKI. The operational definition of acute kidney injury was made if glomerular filtration (GFR) decrease by more than $50 \%$ or double increase of serum creatinine level from baseline as per the 'RIFLE GFR' criteria. ${ }^{12}$ Subjects having simultaneous infections other than urinary tract infection (UTI) or receiving chronic dialysis was excluded.

Base line demographics like age, gender, and educational level were recorded. The recorded risk factors were hypertension, diabetes mellitus (DM), Hepatitis, AIDS, cardiac diseases, cerebrovascular disease, neoplasm, and medications (antihypertensive, nephrotoxic agents, and non-steroidal anti-inflammatory drugs).All the admitted patients were treated with antibiotics according to the standard protocol.

Data analysis was done in SPSS version 23. Descriptive statistics like mean and standard deviation was calculated for numerical variables like age, Systolic blood pressure (BP), Diastolic BP, Serum creatinine, and Baseline eGFR. We use chi-square test for categorical variables and for continuous variable we used student test. Frequency was computed for categorical variable DM, hypertension, and cause of AKI etc. Multivariate logistic regression was carried using $\mathrm{AKI}$ as dependent variable and risk factors as independent variables. $\mathrm{P}=0.05$ was considered significant.

\section{RESULTS}

The male were $87(43.5 \%)$ and female were $113(56.5 \%)$. The mean age of the study was $45.19 \pm 16.2$ years. The mean systolic blood pressure (BP), diastolic BP, serum creatinine, baseline glomerular filtration rate was $130.3 \pm 15.03 \mathrm{~mm} \mathrm{Hg}, 90.3 \pm 15.04 \mathrm{~mm} \mathrm{Hg}$, $2.47 \pm 1.19 \mathrm{mg} / \mathrm{dl}$ and $67.98 \pm 20.5 \mathrm{~mL} / \mathrm{min} / 1.73 \mathrm{~m}^{2}$ respectively. In overall study the nephrotoxic agents, diabetes mellitus, hypertension, upper respiratory tract infection, septic shock were found in $77(38.55), 59(29.5 \%), 54(27.0 \%), 121(60.5 \%)$, and $21(10.5 \%)$ cases respectively.

The frequency of diabetes mellitus $(P=.008)$, hypertension $(P<.001)$, upper respiratory tract infection $(P=.030)$, septic shock $(P=.011)$ and nephrotoxic agents $(P=.013)$ were more in cases than controls and results were statistically significant. (Table 1)

The mean age in cases $(51.57 \pm 15.73$ years) was higher than controls (38.81 \pm 14.2 years) and results were very high statistically significant $(P<0.001 ; 95 \% \mathrm{Cl}=8.5,16.9)$. Similarly the serum creatinine and baseline eGFR was very highly statistically significantly more in cases than controls $(P<0.001)$. However, the results for systolic and diastolic BP was not significant ( $P>0.05)$. (Table 2)

Table 3 shows the multivariate logistic decrease model for factors related to AKI using the control (patients with no $\mathrm{AKI}$ ) as a reference category. Coronary artery disease, upper respiratory tract infection, septic shock and systolic blood pressure were not significant risk factors for AKI after adjusting with logistic regression model as covariate $(P>0.05)$. Positive affect was found for AKI with increasing age $(O R=1.06 ; 95 \%$ $\mathrm{Cl}=1.017,1.105)$, serum creatinine (OR $=5.9 ; 95 \%$ $\mathrm{Cl}=2.85,12.55)$. There was 5.9 odds increase in serum creatinine in patients with $\mathrm{AKI}$ as compared to controls. There was negative association between GFR and AKI (OR=0.92; 95\% Cl=0.888, 0.953). 
Significant Risk Factors for Acute Kidney Injury- A Case Control Study

Table 1. Comparison of categorical risk factors in patients with and without AKI

\begin{tabular}{|c|c|c|c|c|c|c|}
\hline & \multicolumn{4}{|c|}{ Acute Kidney Injury } & \multirow{3}{*}{ P-Value } \\
\hline & & \multicolumn{2}{|c|}{ No } & \multicolumn{2}{|c|}{ Yes } & \\
\hline & & $\mathbf{N}$ & $\%$ & $\mathbf{N}$ & $\%$ & \\
\hline \multirow{2}{*}{ Gender } & Male & 47 & 54 & 40 & 46 & \multirow{2}{*}{.318} \\
\hline & Female & 53 & 46.9 & 60 & 53.1 & \\
\hline \multirow{2}{*}{$\begin{array}{l}\text { Diabetes } \\
\text { Mellitus }\end{array}$} & Yes & 21 & 35.6 & 38 & 64.4 & \multirow{2}{*}{.008} \\
\hline & No & 79 & 56 & 62 & 44 & \\
\hline \multirow{2}{*}{ Hypertension } & Yes & 9 & 14.3 & 54 & 85.7 & \multirow{2}{*}{$<.001$} \\
\hline & No & 91 & 66.4 & 46 & 33.6 & \\
\hline \multirow{2}{*}{$\begin{array}{l}\text { Upper } \\
\text { Respiratory tract } \\
\text { infection }\end{array}$} & Yes & 32 & 40.5 & 47 & 59.5 & \multirow{2}{*}{.030} \\
\hline & No & 68 & 56.2 & 53 & 43.8 & \\
\hline \multirow{2}{*}{ Septic Shock } & Yes & 5 & 23.8 & 16 & 76.2 & \multirow{2}{*}{.011} \\
\hline & No & 95 & 53.1 & 84 & 46.9 & \\
\hline \multirow{2}{*}{$\begin{array}{l}\text { Nephrotoxic } \\
\text { agents }\end{array}$} & Yes & 30 & 39 & 47 & 61 & \multirow{2}{*}{.013} \\
\hline & No & 70 & 56.9 & 53 & 43.1 & \\
\hline
\end{tabular}

"chi-square test

Table 2. Comparison of continuous risk factors in patients with and without AKI

\begin{tabular}{|c|c|c|c|c|}
\hline & $\begin{array}{l}\text { acute kidney } \\
\text { injury (AKI) }\end{array}$ & Mean \pm SD & P-Value ${ }^{*}$ & $95 \% \mathrm{Cl}$ \\
\hline \multirow{2}{*}{ Age } & Yes & $51.57 \pm 15.73$ & \multirow{2}{*}{$<0.001$} & \multirow{2}{*}{$8.5,16.9$} \\
\hline & No & $38.81+14.2$ & & \\
\hline \multirow{2}{*}{ Systolic BP } & Yes & $131.7 \pm 16.4$ & \multirow{2}{*}{.189} & \multirow{2}{*}{$-1.38,6.98$} \\
\hline & No & $128.9+13.48$ & & \\
\hline \multirow{2}{*}{ Diastolic BP } & Yes & $91.7+16.4$ & \multirow{2}{*}{.179} & \multirow{2}{*}{$-1.37,6.99$} \\
\hline & No & $88.9+13.48$ & & \\
\hline \multirow{2}{*}{$\begin{array}{l}\text { Serum } \\
\text { creatinine }\end{array}$} & Yes & $3.21 \pm 0.951$ & \multirow{2}{*}{$<0.001$} & \multirow{2}{*}{$1.22,1.74$} \\
\hline & No & $1.735 \pm 0.929$ & & \\
\hline \multirow{2}{*}{$\begin{array}{l}\text { Baseline eGFR } \\
(\mathrm{mL} / \mathrm{min} / 1.73 \\
\left.\mathrm{m}^{2}\right)\end{array}$} & Yes & $55.23 \pm 16.93$ & \multirow[b]{2}{*}{$<0.001$} & \multirow[b]{2}{*}{$-30.01,-21.0$} \\
\hline & No & $80.74 \pm 15.34$ & & \\
\hline
\end{tabular}

"Independent $\mathrm{t}$ test

Table 3. Multivariate logistic regression model for factors related to "acuite kidney injury"

\begin{tabular}{|l|c|c|c|}
\hline \multicolumn{1}{|c|}{ Covariate } & $\boldsymbol{\beta}$ & P-value & OR (95\% Cl) \\
\hline Coronary artery disease & 0.437 & 0.793 & $1.54(0.06,40.19)$ \\
\hline Upper Respiratory tract infection & -0.254 & 0.668 & $0.776(0.24,2.47)$ \\
\hline Septic shock & -0.477 & 0.612 & $0.621(0.098,3.92)$ \\
\hline Hypertension & -2.823 & $<0.001$ & $0.059(0.014,0.259)$ \\
\hline Diabetes & -1.46 & 0.044 & $0.232(0.056,0.961)$ \\
\hline Age & 0.058 & 0.006 & $1.06(1.017,1.105)$ \\
\hline Systolic blood pressure & 0.01 & 0.555 & $1.01(0.97,1.045)$ \\
\hline Serum Creatinine & 1.789 & $<0.001$ & $5.9(2.85,12.55)$ \\
\hline Glomerular filtration rate & -0.084 & $<0.001$ & $0.92(0.888,0.953)$ \\
\hline Constant & 0.477 & 0.891 & 1.611 \\
\hline
\end{tabular}




\section{DISCUSSION}

This case control study was conducted to ascertain the risk factors for 'acute kidney injury (AKI)'. Multivariate logistic regression analysis was done to control confounders in the study and to determine associated risk factors for AKI. Our findings showed that the frequency of diabetes mellitus $(P=.008)$, hypertension $(P=<.001)$, upper respiratory tract infection $(P=.030)$, septic shock $(P=.011)$ and nephrotoxic agents $(P=.013)$ were more in cases than controls and results were statistically significant. After controlling confounders through regression analysis our results showed that increasing age $(O R=1.06)$, serum creatinine $(\mathrm{OR}=5.9)$ and $\mathrm{GFR}(\mathrm{OR}=0.92)$ were significant risk factor for 'acute kidney injury $(\mathrm{AKI})^{\prime}$.

In this study we include the patients with UTI which can lead to sepsis and septic shock. UTI can cause 'acute kidney injury (AKI)'. ${ }^{12}$ Earlier investigations reported that hypotension, hypovolemia, the use of nephrotoxic agents, sepsis, urinary obstruction, contrast media were risk factors in UTI affected subjects for AKI. ${ }^{13,} 14$ Hsiao et al. ${ }^{12}$ conducted an investigation to assess the risk factors of Acute Kidney Injury in UTI patients. They reported that associated risk factors for AKI were older age, upper UTI, diabetes mellitus (DM), lower baseline eGFR and a febrile during hospitalization. These results are in favor of our study except a febrile during hospitalization.

Our findings showed that that increasing age $(O R=1.06)$ was significant risk factor for 'acute kidney injury $(\mathrm{AKI})$ '. Hsiao et al. ${ }^{12}$ reported that an odd ratio of 1.20 for increasing age with $\mathrm{AKI}$ in their multivariate logistic regression. These results are in consistent with our study. The reason for this association can due to the fact that in elderly there is high prevalence of UTI as compared younger population. ${ }^{15}$ In addition, age-related decrease in glomerular filtration rate may cause elder populations at more risk for $\mathrm{AKI}^{16}$

Our findings showed that increase in serum creatinine was associated with 5.9 time increase risk for AKI (OR=5.9). Serum creatinine level is normally used in clinical practice to assess the renal function. The value of serum creatinine above one shows the dysfunction of kidney. This showed that renal dysfunction is major cause of 'acute kidney injury (AKI)'. Serum creatinine is also used directly for diagnosing AKI. ${ }^{12}$ Similarly results were reported by Hsiao et al. ${ }^{12}$

The current study showed that less GFR was protective for 'acute kidney injury (AKI)' with odd ratio of 0.92 . This shows that in 'acute kidney injury (AKI)' the GFR value become higher. Similar results were found by Hsiao et $\mathrm{al}^{12}$. Hsu et al. $^{17}$ conducted a study on chronic kidney disease patients having hospital acquired acute kidney injury managed with and without dialysis, and they reported that if eGFR is less than 60 $\mathrm{ml} /$ minutes $/ 1.73 \mathrm{~m}^{2}$, there is higher risk of AKI. Our findings showed that renal dysfunction (high GFR) have a predictive role for 'acute kidney injury (AKI)' in urinary tract infected patients.

\section{CONCLUSION}

Based on our results it can be concluded that diabetes mellitus, hypertension, upper respiratory tract infection, septic shock and nephrotoxic agents are significant risk factors for AKI. However, after controlling confounding factors through multivariate analysis the increasing age, serum creatinine and low GFR were significant risk factor for 'acute kidney injury (AKI)'.

\section{REFERENCES}

1. Lameire NH, Bagga A, Cruz D, De Maeseneer J, Endre Z, Kellum JA, et al. 'acute kidney injury (AKI)': an increasing global concern. Lancet. 2013;382(9887):170-9.

2. Koza Y. 'acute kidney injury (AKI)': current concepts and new insights. J Inj Violence Res. 2016;8(1):58-62.

3. Jha V, Parameswaran S. Community-acquired 'acute kidney injury (AKI)' in tropical countries. Nat Rev Nephrol. 2013;9(5):278-90.

4. Bagasha $P$, Nakwagala $F$, Kwizera A, Ssekasanvu E, Kalyesubula R. 'acute kidney injury (AKI)' among adult patients with sepsis in a low-income country: clinical patterns and short-term outcomes. Bio Med Cent Nephrol. 2015;16(1):4-9.

5. Mori T, Shimizu T, Tani T. Septic acute renal failure. Acute Blood Purification: Karger Publishers; 2016. p. 40-6.

6. Plataki M, Kashani K, Cabello-Garza J, Maldonado F, Kashyap R, Kor DJ, et al. Predictors of 'acute kidney injury (AKI)' in septic shock patients: an observational cohort study. Clin JAm Soc Nephrol. 2011;6(7):1744-51.

7. Thakar CV, Christianson A, Almenoff P, Freyberg R, Render ML. Degree of 'acute kidney injury (AKI)' before dialysis initiation and hospital mortality in critically ill patients. Int J Nephrol. 2013;2013(4):34-40.

8. Hsu C-y, Chertow GM, McCulloch CE, Fan D, Ordoñez JD, Go AS. Nonrecovery of kidney function and death after acute on chronic renal failure. Clin $\mathrm{J} \mathrm{Am} \mathrm{Soc}$ Nephrol. 2009;4(5):891-8.

9. Kaddourah A, Basu RK, Bagshaw SM, Goldstein SL. Epidemiology of 'acute kidney injury (AKI)' in critically ill children and young adults. New England J Med. 2017;376(1):11-20. 
10. Ponce $D$, Balbi A. 'acute kidney injury (AKI)': risk factors and management challenges in developing countries. Int J Nephrol Renovas Dis. 2016;9:193-200.

11. Krishnamurthy S, Mondal N, Narayanan P, Biswal N, Srinivasan S, Soundravally R. Incidence and etiology of 'acute kidney injury (AKI)' in southern India. Ind J Pediatr. 2013;80(3):183-9.

12. Hsiao C-Y, Yang H-Y, Hsiao M-C, Hung P-H, Wang M-C. Risk factors for development of 'acute kidney injury (AKI)' in patients with urinary tract infection. PloS one. 2015;10(7):e0133835.

13. Kooman JP, Barendregt JN, Van der Sande FM, Van Suylen RJ. Acute pyelonephritis: a cause of acute renal failure? Neth J Med. 200;57:1851-8.

14. Nahar A, Akom M, Hanes D, BrigliaA, Drachenberg CB, Weinman EJ. Pyelonephritis and acute renal failure Am J Med Sci. 2004;328:121-3.

15. Boscia JA, Kaye D. Asymptomatic bacteriuria in the elderly. Infect Dis Clin NorthAm. 1987;1:89390-5.

16. Chawla LS, Eggers PW, Star RA, Kimmel PL. 'acute kidney injury (AKI)' and chronic kidney disease as interconnected syndromes. N Engl J Med. 2014; 371: 586-91.

17. Hsu C, Ordonez J, Chertow G, Fan D, McCulloch C, GoA. The risk of acute renal failure in patients with chronic kidney disease. Kidney Int. 2008;74(1):101-7.
DATA SHARING STATEMENT: The data that support the findings of this study are available on request from the corresponding author. The data are not publicly available due to privacy or ethical restrictions.

CONFLICT OF INTEREST: Authors declared no conflict of interest.

GRANTED SUPPORT AND FINANCIAL DISCLOSURE: Nil

AUTHOR'S CONTRIBUTION
Following authors have made substantial contributions to
the manuscript as under
Samiullah, Ayub M: Concept and design of study,
Collection of data, statistical analysis
Saeed M, Aminullah: Writing of manuscript, critical review
of manuscript
Rashieed AU: Analysis and interpretation of data,
statistical analysis
Daheem S: $\quad$ Data collection, bibliography
Authors agree to be accountable for all aspects of the work
in ensuring that questions related to the accuracy or
integrity of any part of the work are appropriately
investigated and resolved.

